\title{
Exercise-induced Thoracic Outlet Syndrome and Concomitant Osteomyelitis in Cervical Rib with a Possible Familial Origin, a Case Report.
}

Mohammadshah Isam Gul

Hamad Medical Corporation

Ammara Bint I Bilal ( $\square$ ammarabint_i_bilal@hotmail.com )

Hamad Medical Corporation https://orcid.org/0000-0002-0219-1678

Fateen Ata

Hamad Medical Corporation

Renan E Ibrahem

Hamad Medical Corporation

Muhammad I Danjuma

Hamad Medical Corporation

\section{Case report}

Keywords: Cervical rib, accessory rib, osteomyelitis, familial

Posted Date: April 9th, 2021

DOl: https://doi.org/10.21203/rs.3.rs-403083/v1

License: (9) This work is licensed under a Creative Commons Attribution 4.0 International License. Read Full License 


\section{Abstract}

\section{Background}

Cervical ribs are a rare and usually asymptomatic occurrence. Most are identified incidentally by Roentgenogram (X-rays). However, occasionally they can cause nerve impingements and compressive symptoms. In cervical ribs, osteomyelitis secondary to trauma is unheard of. We report such a case made more interesting by the familial presence of bilateral cervical ribs in 2 generations. This indicates a possible familial origin.

\section{Case presentation}

A 26-year-old woman known to have fibromyalgia presented with left shoulder pain and fever episodes. She was recently discharged from intensive physiotherapy for fibromyalgia management, but the pain aggravated instead of getting better unilaterally in the left arm after discharge. Electric shock-like sensations and reduced strength accompanied this. An evaluation revealed leukocytosis and S. aureus positive blood and urine cultures along with bilateral complete cervical ribs showing pseudoarthrosis of anterior left cervical rib. A collection was noted in the left scalene muscle near the pseudoarthrosis accompanying focal edema suggesting osteomyelitis. Transthoracic echocardiogram (TTE) ruled out infective endocarditis. Thoracic surgery recommended conservative management, and appropriate antibiotics were given, which resulted in the negative culture on day 6 . The patient was discharged on appropriate analgesia, and her pain was significantly improved on discharge.

\section{Conclusions}

This is the first reported case of osteomyelitis in the cervical rib. This report also highlights the possibility of cervical ribs having a familial origin. Additionally, as in our patient, certain exercises can be a risk factor for inducing TOS in patients with cervical rib.

\section{Background}

Cervical ribs, commonly addressed as accessory or neck ribs, are rare congenital supernumerary ribs arising from the 7th vertebra. This anatomic variant's prevalence is believed to be underreported as only symptomatic cases are brought to attention, whether unilateral or bilateral [1]. A gender preference is observed, with the condition being twice as prevalent in women as men [2]. Thoracic outlet syndrome (TOS) is caused by the compression of the subclavian artery/vein and/or the brachial plexus as they traverse through the thoracic outlet. TOS development is the leading clinical picture of discovery and morbidity associated with cervical ribs[1]; such ribs are large and frequently fused to the first rib. This placement also predisposes to aneurysm formation or thrombosis [3].

Cervical ribs have been associated with mutations in the HOX genes responsible for constructing axial skeleton patterns [4]. Owing to their rarity, it has been challenging to establish a familial pattern. Taku 
Suzuki et al. have previously presented clinical images of bilateral cervical ribs in a mother and daughter, hinting towards genetic predisposition, although the available data in the literature is trivial [5]. In the early 20th century, a family was reported in which seven out of 11 members had cervical ribs, parents having unilateral, and five children with bilateral cervical ribs [4]. Osteomyelitis of ribs (OR) is rare and is usually associated with tuberculosis wherever seen [6]. Rare cases have been reported of nontuberculous $\mathrm{OR}$, but no current literature exists regarding cervical rib osteomyelitis. We present an interesting case of bilateral cervical ribs in a patient with fibromyalgia in which TOS was triggered by trauma, further complicated by osteomyelitis, and thorough history revealed a familial pattern of cervical ribs.

\section{Case Presentation}

A 26-year-old Pakistani female presented to the emergency department with a five-day history of gradually increasing left shoulder pain for one week. She also had two episodes of fever at home, for which she took paracetamol. The patient was a known case of fibromyalgia, which was diagnosed in 2018. Her disease was stable on amitriptyline and gabapentin with physiotherapy. She also had postural tachycardia syndrome, for which she was taking bisoprolol $2.5 \mathrm{mg}$ daily.

She was discharged a week ago from the rehabilitation institute for physiotherapy, where she stayed for a few days as a part of her fibromyalgia management. During her stay, the patient developed left shoulder pain as a result of intensive physiotherapy. The pain was managed with tramadol oral $50 \mathrm{mg}$ thrice daily as needed (PRN) for two days and celecoxib $200 \mathrm{mg}$ twice daily for five days. She was discharged from the rehabilitation center on celecoxib.

The intensity of the pain at discharge from physical therapy rehabilitation was $3 / 10$, which gradually increased to $8 / 10$. She described it as an electric shock-like sensation radiating from her left shoulder to the left hand. The patient also has an on and off burning sensation in the upper left arm. There was minimal movement in the left arm due to pain, which was not responding well to the analgesia. She denied cough, running nose, headache, chest pain, palpitation, abdominal pain, diarrhea, constipation, dysuria, urgency, or increase in frequency. Upon physical examination, the patient was afebrile $\left(37.2^{\circ} \mathrm{C}\right)$, with a heart rate of 96 beats/min, respiratory rate of $18 / \mathrm{min}$, and blood pressure of $109 / 73 \mathrm{mmHg}$ (which was normal for her). She was maintaining normal saturation.

As she had two fever episodes at home, infection was thought of as a possibility, and an infectious disease workup was sent. Results showed leukocytosis of $22.1 \times 10^{3} / \mathrm{ul}$ with absolute neutrophil count of $18.2 \times 10^{3} / \mathrm{ul}$, normocytic anemia with hemoglobin of $11.6 \mathrm{gm} / \mathrm{dL}$ (on discharge it was $13.2 \mathrm{gm} / \mathrm{dL}$ ) and elevated platelets of $672 \times 10^{3} / \mathrm{ul}$ (on discharge: $255 \times 10^{3} \mathrm{ul}$ ). She had a raised $\mathrm{C}$ reactive protein (CRP) level $(118 \mathrm{mg} / \mathrm{L})$ with a normal procalcitonin and lactic acid. Her kidney functions and electrolytes were within the normal range, and she had mildly elevated liver functions (alanine aminotransferase:139U/L, aspartate aminotransferase: $38 \mathrm{U} / \mathrm{L}$, and alkaline phosphatase $286 \mathrm{U} / \mathrm{L}$ ). Blood and urine bacterial cultures were sent, and the patient was started on ceftriaxone intravenously (IV) $2 \mathrm{gm}$ daily, tramadol $50 \mathrm{mg}$ oral PRN q8hrs, and regular paracetamol $1 \mathrm{gram}$ oral q6hrs. 
Before admission, she had visited a private clinic where an ultrasound of the left shoulder was done, which was reported as marked heterogenicity of the scalene muscle of left with surrounding soft tissue edema and irregular collection $(10 \mathrm{ml})$ towards the root of the neck. X-ray of the chest $(\mathrm{CXR})$ and left shoulder showed no fracture; however, it revealed bilateral complete cervical ribs (Fig. 1a and 1b).

Given the findings of the ultrasound with a suspicion of possible infective collection (due to the fever episodes) or hematoma (as she had a drop in hemoglobin level), a computerized tomography (CT) scan of the neck and thorax was performed. It revealed a bulky appearing left scalene muscle $(6 \times 3 \mathrm{~cm}$ in maximum) with necrotic component or hematoma measuring $21 \times 18 \mathrm{~mm}$, associated with fat stranding and left supraclavicular lymph nodes. It also detailed the previously seen cervical ribs, with the left cervical rib deformed anteriorly and fused with the left first rib (pseudoarthrosis) (Fig. 2).

Blood and urine cultures came positive for Staphylococcus aureus (S. aureus) by day 3. Her antibiotics were changed to cefazolin intravenous (IV) q8hrs and one shot of vancomycin 1.5gm IV (while awaiting S. aureus sensitivity, which eventually turned to be methicillin-sensitive).

Magnetic resonance imaging (MRI) of the left shoulder was performed to evaluate better the necrotic component seen previously in the CT scan and rule out septic arthritis as a possible diagnosis. The shoulder joint appeared normal. The collection appeared to be related to the left cervical rib's pseudoarthrosis with the first deformed rib. It also showed focal low-grade marrow edema involving the cervical rib. The findings were suspicious of osteomyelitis of the left cervical rib's distal end (Fig. 3).

Thoracic surgery was consulted for the possibility of drainage; however, conservative treatment was recommended with an outpatient follow-up.

A transthoracic echocardiogram was done to rule out infective endocarditis; it showed no vegetation. Repeated blood cultures were negative by day 6 . She completed one week of cefazolin IV q8hrs. Her pain improved, and she was discharged on analgesics (paracetamol/orphenadrine 1000mg q8hrs, diclofenac sodium cream q12hrs, and naproxen $250 \mathrm{mg} \mathrm{q12hrs} \mathrm{PRN)} \mathrm{for} \mathrm{five} \mathrm{more} \mathrm{days} \mathrm{and} \mathrm{Cephalexin} 1 \mathrm{gm}$ q8hrs for another five weeks to complete the total duration of 6 weeks for the osteomyelitis.

\section{Discussion}

Osteomyelitis is a medical emergency characterized by infection of the bones [7]. The most commonly involved bones are long bones of arms and legs in children, whereas, in adults, the most commonly involved bones are pelvic bones, spine, and lower limbs [8]. It may be caused by a multitude of microorganisms, including bacteria, viruses, parasites, and fungi. However, the most common association is with pyogenic bacteria and mycobacteria. Among the cases of pyogenic osteomyelitis, S. aureus is found in $80-90 \%$ cases, whereas Staphylococcus epidermidis (which is the most prevalent skin flora) primarily tends to infect medical devices, including implants and catheters [9]. 
Rib involvement for osteomyelitis is rare [10], with osteomyelitis of the cervical ribs in an adult not reported before, to the best of our knowledge. It is unknown whether an accessory rib inherently has increased, decreased, or similar predisposition to developing an infection of the bone and whether any specific bacteria are more likely to infect such anatomic variants. In our case, the patient developed S. aureus osteomyelitis. This area of cervical rib complications needs further exploration.

Cervical ribs narrow the boundaries of the inter scalene triangle forming the inferior border before the first rib [11]. This predisposes to the development of TOS, which can be neurogenic or vascular. This compression neuropathy is one of the most challenging neuropathies for neurosurgeons as no definitive guidelines exist as to when should the supernumerary rib be surgically excised. A variable clinical presentation arises with complete vs. incomplete cervical ribs as vascular symptoms are almost exclusive to complete cervical ribs[1].

Without any acute neurological or vascular manifestation in symptomatic patients, treatment depends on the corresponding manifestations. All cases are treated conservatively by pain killers such as nonsteroidal anti-inflammatory drugs (NSAIDs) and muscle relaxants and emphasizing lifestyle changes. Physiotherapy is encouraged to strengthen the muscles around the shoulder girdle. Surgery is considered if all conservative measures have failed, and it is the treating physician's clinical decision [4].

Our patient developed radiating neuropathic pain following rehabilitation exercise for fibromyalgia, indicating a possible trigger of TOS. The question arises if such patients should be recommended to exercise with caution or if they should avoid certain movements that can narrow the interscalene triangle further? Again this area is unexplored yet contributes significantly to avoidable morbidity in patients with cervical ribs.

Although not established, the familial origin of cervical ribs has been proposed as a possibility. A metaanalysis has demonstrated variability in the prevalence of cervical ribs with respect to geographical and ethnic diversities, supporting a crucial role of genetics [1]. Furthermore, clinically, the presence of cervical ribs in families has long been accepted; however, the inheritance patterns remain unknown. In current literature, a study has suggested autosomal dominant inheritance as they found nine members spanning three generations of a South African family [12]. In our case, the patient's brother and mother both have cervical ribs (Fig. 4), whereas further family history is undocumented. The brother was identified to have cervical ribs by X-rays in Pakistan; however, a repeated CXR was not performed in Qatar.

\section{Conclusion}

Understanding the predispositions and consequences of cervical ribs is vital to anticipate and effectively manage the complications associated with this genetic anomaly. Familial origin has been suggested multiple times, but no convincing evidence exists as it is still an under-explored area in medicine. Trauma, overuse, and poor posture are the leading causes that predispose individuals to symptoms. More extensive studies focusing on avoidable triggers are needed since the development of TOS confers a substantial decrease in quality of life. The pathophysiology behind cervical rib osteomyelitis remains 
unexplored, but in our case, since it was S.aureus induced, we can conclude that it possibly follows the same trends as any other osteomyelitis. However, further research is required to make evidence-based conclusions regarding:

a) Is there a familial origin of cervical ribs? If so, what is the inheritance pattern?

b) What are the avoidable triggers, e.g., exercises that may narrow the scalene triangle further to induce TOS in patients with asymptomatic cervical ribs?

c) Is osteomyelitis in cervical ribs any different from osteomyelitis in other ribs?

\section{Abbreviations}

Thoracic outlet syndrome (TOS)

Staphylococcus aureus (S.aureus)

Chest X-ray (CXR)

Non-steroidal anti-inflammatory drugs (NSAIDs)

As needed (PRN)

Computerized tomography (CT)

Magnetic resonance imaging (MRI)

Intravenous (IV)

C reactive protein (CRP)

Osteomyelitis of ribs (OR)

Transthoracic echocardiogram (TTE)

Roentgenogram (X-rays)

\section{Declarations}

\section{Acknowledgments:}

The publication of this article was funded by the Qatar National Library.

\section{Ethics approval and consent to participate.}


Ethical approval for this study was obtained from Medical Research Center (MRC) Qatar (MRC-01-20577).

\section{Consent for publication}

Written consent was taken from the patient and her mother for publication of data and accompanying images.

\section{Availability of data and materials}

Not applicable

\section{Competing interests}

The authors declare that they have no competing interests.

\section{Funding}

This study was not funded.

\section{Authors' contributions}

(highlighted authors share co-first authorship due to equal contribution)

MG: Literature review, Manuscript writing, obtained written consent

AB: Literature review, Manuscript writing, radiological image selection

RA: Radiology workup of the patient, finalization of the images, figure legends

FA: Literature review, Critical review, and revisions in the manuscript

MD: Literature review, Critical review, and revisions in the manuscript

All authors read and approved the final version of this manuscript.

\section{References}

1. Henry BM, Vikse J, Sanna B, Taterra D, Gomulska M, Pękala PA, Tubbs RS, Tomaszewski KA: Cervical Rib Prevalence and its Association with Thoracic Outlet Syndrome: A Meta-Analysis of 141 Studies with Surgical Considerations. World Neurosurg 2018, 110:e965-e978. 
2. Viertel VG, Intrapiromkul J, Maluf F, Patel NV, Zheng W, Alluwaimi F, Walden MJ, Belzberg A, Yousem DM: Cervical ribs: a common variant overlooked in CT imaging. AJNR Am J Neuroradiol 2012, 33(11):2191-2194.

3. Chang KZ, Likes K, Davis K, Demos J, Freischlag JA: The significance of cervical ribs in thoracic outlet syndrome. J Vasc Surg 2013, 57(3):771-775.

4. Spadliński $Ł$, Cecot T, Majos A, Stefańczyk L, Pietruszewska W, Wysiadecki G, Topol M, Polguj M: The Epidemiological, Morphological, and Clinical Aspects of the Cervical Ribs in Humans. BioMed Research Internationa/ 2016, 2016:8034613.

5. Suzuki T, Kimura H, Matsumura N, Sato K, Iwamoto T: Familial Cervical Rib. Journal of Clinical Images 2020, 3(1):1074.

6. Mohanty D, Agrawal V, Jain BK, Gupta R, Rathi V, Gupta A: Osteomyelitis of the ribs: a strategy for prompt diagnosis and effective management. Trop Doct 2008, 38(4):239-241.

7. Schmitt SK: Osteomyelitis. Infect Dis Clin North Am 2017, 31(2):325-338.

8. Waldvogel FA, Medoff G, Swartz MN: Osteomyelitis: a review of clinical features, therapeutic considerations and unusual aspects. N Engl J Med 1970, 282(4):198-206.

9. Birt MC, Anderson DW, Bruce Toby E, Wang J: Osteomyelitis: Recent advances in pathophysiology and therapeutic strategies. J Orthop 2017, 14(1):45-52.

10. Jihad Bishara DG-IMWSMGTSP: Osteomyelitis of the Ribs in the Antibiotic Era. Scandinavian Journal of Infectious Diseases 2000, 32(3):223-227.

11. Tubbs RS, Louis RG, Jr., Wartmann CT, Lott R, Chua GD, Kelly D, Palmer CA, Shoja MM, Loukas M, Oakes WJ: Histopathological basis for neurogenic thoracic outlet syndrome. Laboratory investigation. J Neurosurg Spine 2008, 8(4):347-351.

12. Schapera J: Autosomal dominant inheritance of cervical ribs. Clin Genet 1987, 31(6):386-388.

\section{Figures}



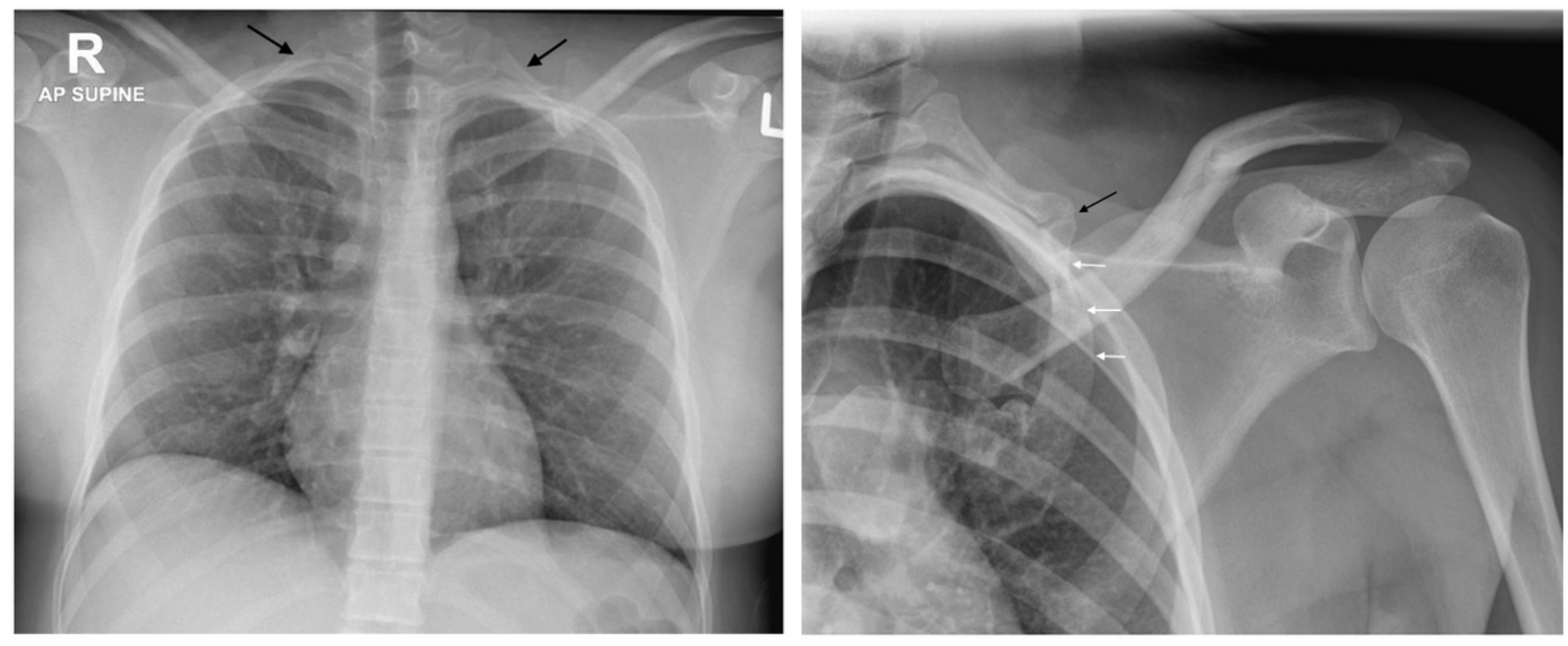

\section{Figure 1}

Left: AP chest radiograph shows bilateral cervical ribs. Right: AP radiograph of the left shoulder shows pseudoarthrosis (black arrow) of the cervical rib with the deformed first rib (white arrows).

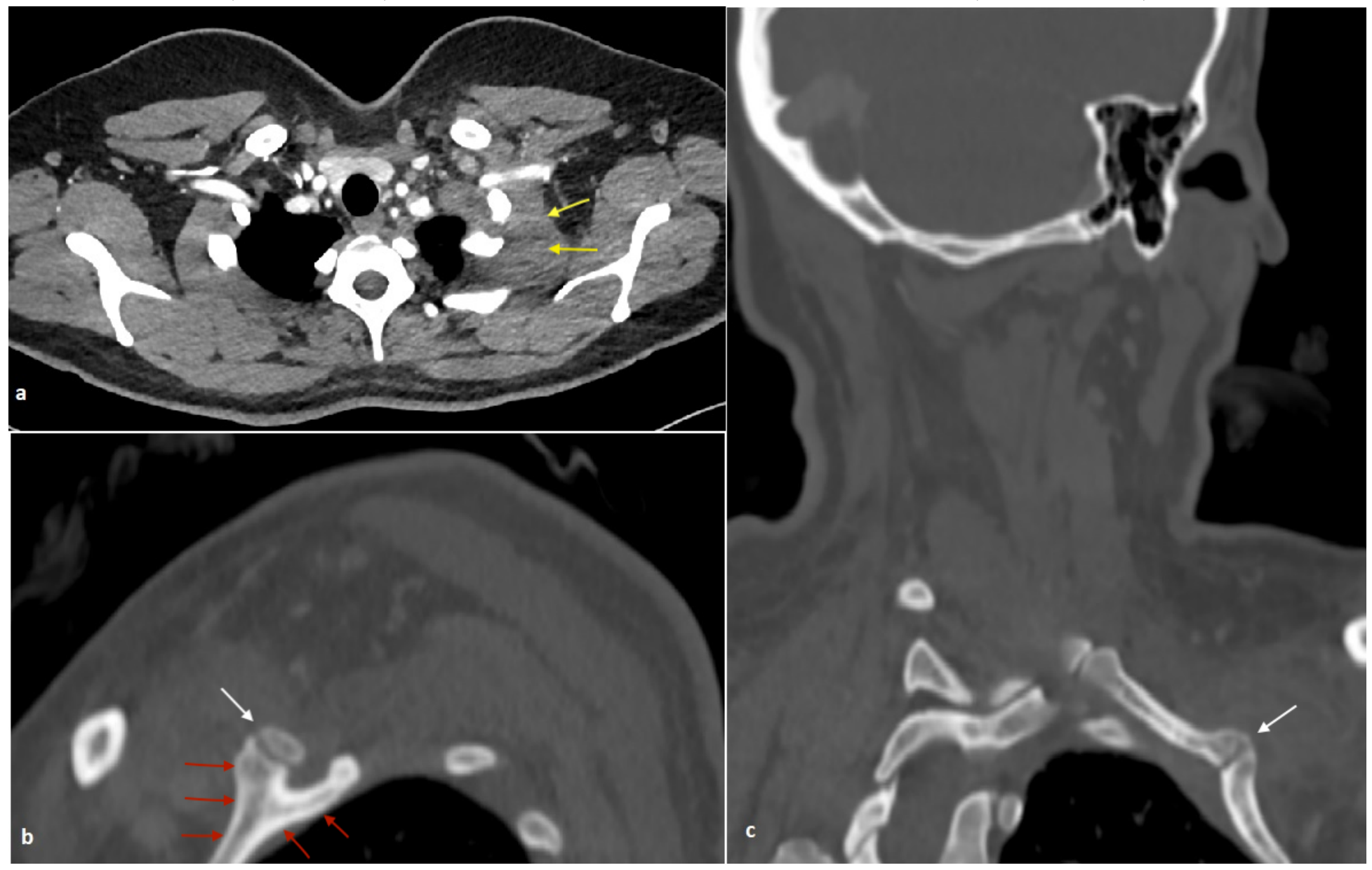

Figure 2 
CT chest. (a) The axial CT image at the apices' level demonstrates thickening of the left scalene muscle with associated soft tissue thickening/hematoma around the left cervical rib's pseudoarthrosis with the first rib (yellow arrows). (b) Sagittal and (c) oblique coronal CT images of the left side of the chest show deformity of the left first rib with bifid appearance anteriorly (red arrows) and associated pseudoarthrosis with the anterior end of the cervical rib (white arrow).

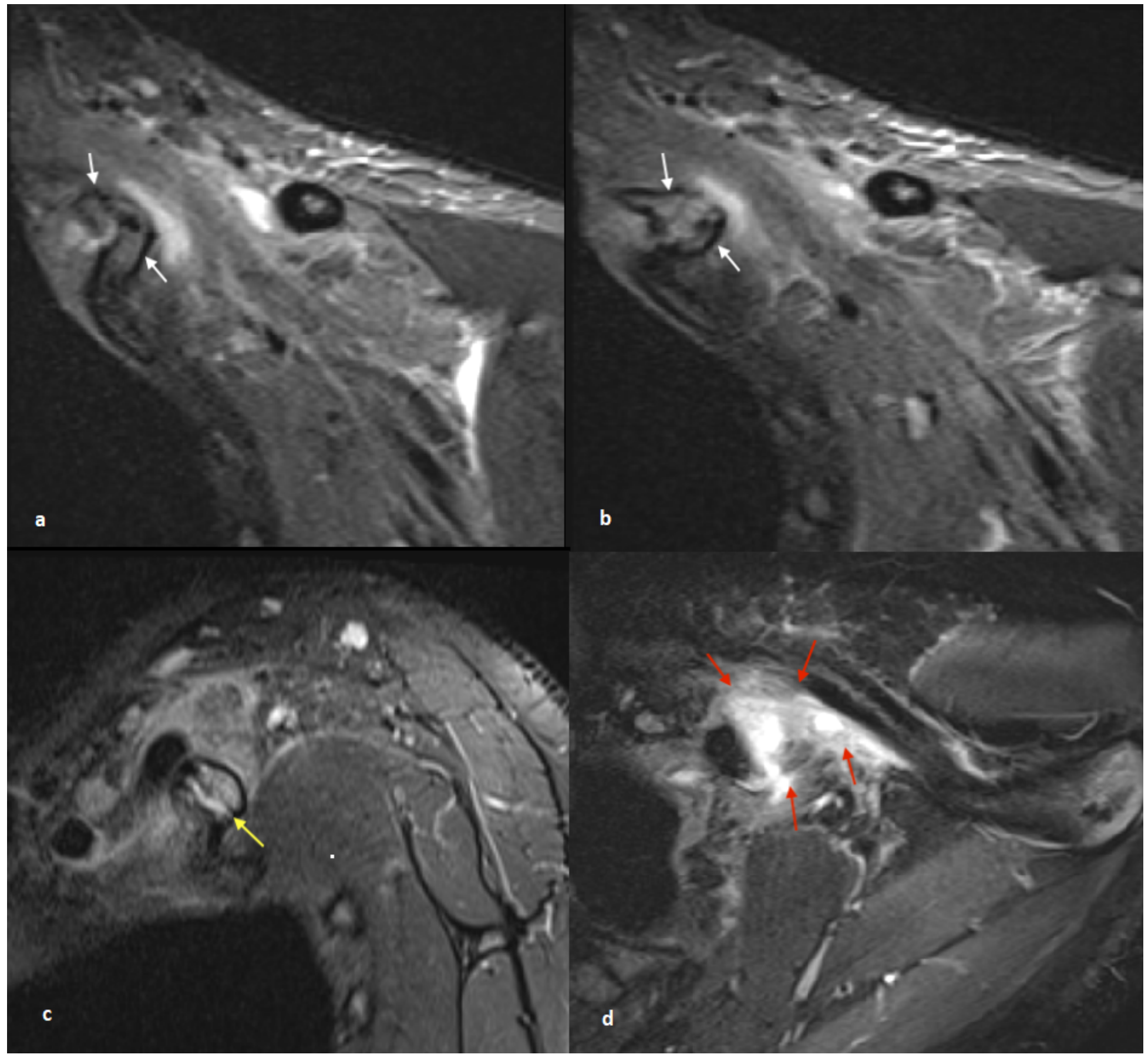

\section{Figure 3}

MRI of the left shoulder. ( $a$ and $b$ ) Coronal STIR images show marrow edema at both ends of the pseudoarthrosis involving the cervical and adjacent first ribs (white arrows). (c) Sagittal and (d) Axial STIR images show a trace of fluid in the pseudoarthrosis region (yellow arrow) with surrounding soft tissue edema associated with small hematoma/ fluid collection (red arrows). 


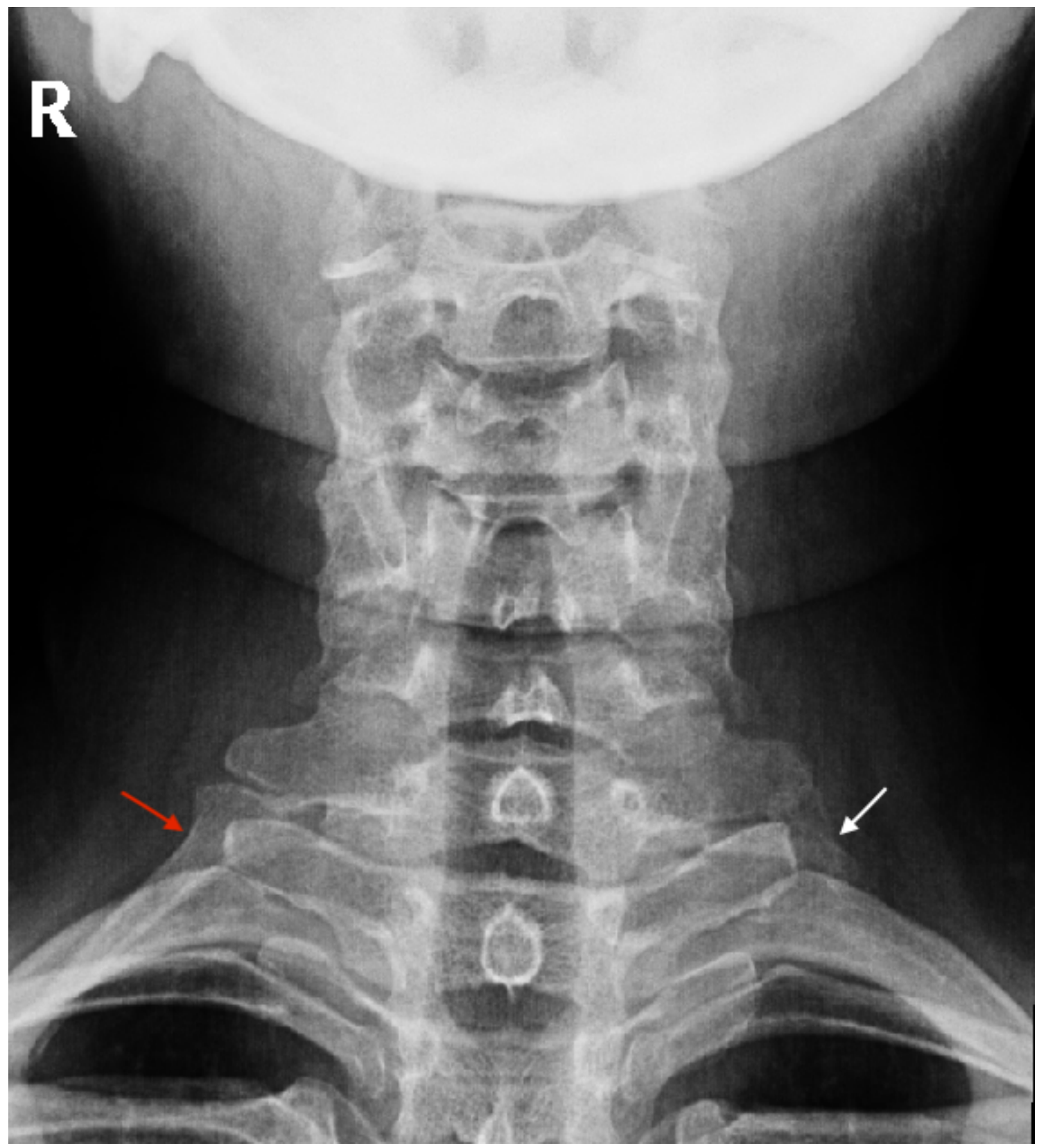

Figure 4

AP cervical radiograph of the patient's mother shows right-sided cervical rib (red arrow) and a rudimentary one on the left side (white arrow).

\section{Supplementary Files}

This is a list of supplementary files associated with this preprint. Click to download.

- CAREChecklist.pdf 